\title{
Factores asociados con la hipertensión no tratada en los adultos mayores: resultados del Estudio Nacional sobre Salud y Envejecimiento en México, 2001
}

\author{
Efrén Melano-Carranza, ${ }^{1}$ Luis Alberto Lasses Ojeda ${ }^{2}$ y José Alberto Ávila-Funes ${ }^{3}$
}

Forma de citar

Melano-Carranza E, Lasses Ojeda LA, Ávila-Funes JA. Factores asociados con la hipertensión no tratada en los adultos mayores: resultados del Estudio Nacional sobre Salud y Envejecimiento en México, 2001. Rev Panam Salud Publica. 2008;23(5):295-302.

RESUMEN Objetivo. Determinar los factores asociados con la falta de adhesión al tratamiento de la hipertensión arterial (HTA) diagnosticada en una muestra representativa de adultos mayores mexicanos que viven en la comunidad.

Métodos. Estudio transversal con 2029 personas de 65 años o más con diagnóstico de HTA, participantes en el Estudio Nacional sobre Salud y Envejecimiento en México realizado en el verano de 2001. La encuesta recabó información sobre algunas características sociodemográficas (edad, sexo, escolaridad, si vivía solo y si realizaba algún trabajo remunerado, entre otras), las enfermedades crónicas, los síntomas depresivos, el deterioro cognoscitivo, el índice de masa corporal, el tabaquismo, el consumo de bebidas alcohólicas y las dificultades para realizar actividades básicas (ABVD) e instrumentales (AIVD) de la vida diaria, entre otras. Se evaluó la asociación entre las variables estudiadas y el autoinforme de hipertensión arterial no tratada (HTNT) mediante análisis de regresión logística simple y multifactorial.

Resultados. De los 2029 participantes, 437 (21,5\%) declararon no seguir tratamiento alguno para controlar la HTA, $1584(78,1 \%)$ afirmaron seguir un tratamiento y $8(0,4 \%)$ no respondieron a esa pregunta. El análisis multifactorial ajustado por posibles variables confusoras (edad, sexo, síntomas depresivos y deterioro cognoscitivo) mostró que solo la baja escolaridad (razón de posibilidades [odds ratio, OR] ajustada =1,70; intervalo de confianza de 95\% [IC95\%]: 1,10 a 2,64; $\mathrm{P}=0,02$ para la escolaridad de 1 a 6 años y OR ajustada =3,32; IC95\%: 2,10 a 5,24; $\mathrm{P}<0,01$ para los no escolarizados), consumir bebidas alcohólicas (OR ajustada= 1,52; IC95\%: 1,14 a 2,03; P = 0,01) y padecer de incontinencia urinaria (OR ajustada =1,61; IC95\%: 1,15 a 2,26; $\mathrm{P}<0,01$ ) resultaron asociadas independientemente con la HTNT.

Conclusiones. La HTNT es un problema frecuente e importante en los adultos mayores en México. Para lograr una mejor adhesión, los médicos deben tomar en cuenta la presencia de incontinencia urinaria, el consumo de bebidas alcohólicas y la baja escolaridad de los pacientes al prescribir o modificar el tratamiento antihipertensivo.

Palabras clave Anciano, hipertensión, tratamiento, falta de cooperación del paciente, México.

Cardiología de Adultos, Instituto Nacional de Cardiología Ignacio Chávez, Ciudad de México, México. 2 Cardiología Geriátrica, Instituto Nacional de Cardiología Ignacio Chávez, Ciudad de México, México.
3 Clínica de Geriatría, Instituto Nacional de Ciencias Médicas y Nutrición Salvador Zubirán, Ciudad de México, México. La correspondencia se debe dirigir a J. Alberto Ávila Funes, Clínica de Geriatría,
Instituto Nacional de Ciencias Médicas y Nutrición Salvador Zubirán. Vasco de Quiroga No. 15, Tlalpan, México, D.F., CP 14000, México. Correo electrónico: avilaf1@quetzal.innsz.mx 
El aumento de la esperanza de vida es una realidad en el mundo. Sin embargo, con el paso de los años se reduce la capacidad física para realizar algunas actividades y aumentan los problemas de salud. En México, por ejemplo, a la población de 65 años o más le corresponde $19,3 \%$ del total de los egresos hospitalarios del Sistema Nacional de Salud (1), cifra que resulta significativa si se considera que la proporción de adultos mayores era de casi 7\% en 2000 (2).

Las enfermedades cardiovasculares constituyen la primera causa de muerte en este sector de la población y la hipertensión arterial (HTA), uno de sus principales factores de riesgo, afecta a más de $60 \%$ de las personas de 65 años o más (2). Según estimados de la Encuesta Nacional de Salud (ENSA) del año 2000, la prevalencia de HTA en la población mexicana es de 30,0\%, pero asciende a más de $50 \%$ en las personas de 50 años o más, por lo que su prevalencia general aumenta a medida que la población envejece (3-5). Según los resultados de la Encuesta Nacional de Salud y Nutrición de los Estados Unidos de América de 2000, la prevalencia de HTA en personas de 60 años o más de ese país es de 65,4\% (6).

La incidencia de las complicaciones asociadas con la HTA crónica en la población general ha disminuido en los últimos 30 años, principalmente debido a que gozan de un mayor acceso al tratamiento (7). Sin embargo, el control inadecuado de la tensión arterial es frecuente en el grupo de los adultos mayores (8), lo que contribuye a elevar el riesgo de infarto del miocardio, insuficiencia cardiaca, enfermedad vascular cerebral y enfermedad renal en este grupo de la población (9-11). Aunque se conoce la importancia de su estricto control, la falta de adhesión al tratamiento prescrito es frecuente y constituye la causa principal del descontrol de la tensión arterial en las personas hipertensas (3). Esto puede deberse en gran parte a la reticencia de los adultos mayores a seguir el tratamiento antihipertensivo con el rigor adecuado, pero también a la falta de insistencia de los médicos. Según algunos estudios, hasta $40 \%$ de los profesionales sanitarios no prescriben tratamiento alguno a los adultos mayores que presentan una tensión arterial sistólica de 140-159 mm $\mathrm{Hg}$ ni prescriben un medicamento adicional al tratamiento antihipertensivo existente con el propósito de mantener la tensión arterial dentro de los valores considerados normales $(12,13)$.

La aplicación de estrategias adecuadas dirigidas a mejorar la adhesión al tratamiento farmacológico antihipertensivo en los adultos mayores puede traer grandes beneficios, tanto a los pacientes como a los sistemas de salud. Sin embargo, para que estas estrategias tengan el éxito esperado deben basarse en la comprensión de las características específicas que inciden en el comportamiento de este grupo de pacientes (8). El objetivo del presente trabajo fue determinar los factores asociados con la falta de adhesión al tratamiento de la HTA diagnosticada en una muestra representativa de adultos mayores mexicanos que viven en la comunidad.

\section{MATERIALES Y MÉTODOS}

Se realizó un estudio transversal de la información obtenida en la primera de las dos vueltas del Estudio Nacional sobre Salud y Envejecimiento en México (ENASEM) en el verano de 2001. Brevemente, el ENASEM es un estudio prospectivo que surge de la cuarta vuelta de la Encuesta Nacional de Empleo y es representativo de los casi 13 millones de mexicanos nacidos antes del $1 .^{\circ}$ de enero de 1951 - ya vivan en zonas urbanas o rurales-y de sus compañeros o parejas independientemente de su edad. Sus objetivos y diseño se publicaron anteriormente (14). En esa primera vuelta del ENASEM se realizaron entrevistas directas a los adultos mayores seleccionados para la muestra o a un sustituto de ellos cuando por algún motivo se encontraban temporalmente ausentes. Los informantes sustitutos debían tener entre 18 y 50 años y vivir en la misma vivienda del adulto mayor seleccionado. El ENASEM es representativo sólo de la población de adultos mayores que no vivían en instituciones, lo cual no es una omisión grave en México ya que, según el II Conteo de Población y Vivienda de
2005, menos de 1\% de la población vivía en residencias o asilos para adultos mayores (15). En total, en el ENASEM se entrevistó a 15230 personas, con una tasa de respuesta de 91,9\%. La encuesta abarcó, entre otros elementos, el autoinforme de los malestares generales, el estado funcional, el tabaquismo, si consumía bebidas alcohólicas, el nivel de acceso a los servicios de salud, los síntomas depresivos, el dolor y el desempeño cognoscitivo; además se tomaron las principales medidas antropométricas. También se recabó información sobre las condiciones de vida que tuvo la persona durante su niñez, la escolaridad, los antecedentes migratorios y maritales y su situación económica (medida a partir de los ingresos por salarios, pensiones y ayudas del gobierno, así como por los bienes de consumo duraderos existentes en el hogar).

Para este estudio, solo se consideraron los participantes de 65 años o más que respondieron afirmativamente a la pregunta "¿alguna vez le ha dicho un médico que tiene hipertensión o tensión arterial alta?"

\section{Variables}

Además de las variables sociodemográficas necesarias para describir la muestra (edad, sexo, escolaridad, si vivía solo y si realizaba algún trabajo remunerado), la encuesta recabó la siguiente información:

\section{Hipertensión no tratada (HTNT).} Cuando la respuesta a la pregunta " $a c$ tualmente, ¿toma algún medicamento para disminuir su tensión arterial?" era "no".

Enfermedades crónicas. Se consideraron como variables categóricas cinco enfermedades (diabetes mellitus, cardiopatía isquémica, enfermedad pulmonar obstructiva crónica, accidente vascular cerebral y artritis), además de la debilidad visual o auditiva y la autopercepción de su estado de salud y de la memoria. Además, la suma de las enfermedades crónicas se utilizó como variable continua. Estas variables se probaron individualmente en el análisis multifactorial. 
Síntomas depresivos. Los síntomas depresivos se evaluaron mediante una versión modificada de la Escala de Depresión del Centro de Estudios Epidemiológicos (CES-D) de los Estados Unidos, la cual ya ha sido validada y aplicada en la población del ENASEM (16). Esta escala se basa en un cuestionario que evalúa algunos síntomas específicos de la depresión geriátrica. El encuestado debía responder "sí" o "no" a nueve preguntas sobre su estado de ánimo en la semana previa. El punto de corte para considerar que había síntomas depresivos era la respuesta afirmativa en al menos cinco preguntas ( $\geq 5$ puntos).

Deterioro cognoscitivo. Se evaluaron los dominios cognoscitivos de atención (detección visual), memoria verbal primaria (repetición de ocho palabras), memoria verbal secundaria (inducción de las ocho palabras), habilidad visoconstruccional (copia de dos figuras) y memoria visual (inducción de las figuras), según una versión reducida de la Evaluación Cognoscitiva Transcultural (17). Se analizó el deterioro cognoscitivo mediante percentiles calculados según el nivel de escolaridad (0, 1 a 4, 5 a 9, y 10 años o más). Se consideró que las personas que quedaron por debajo del percentil 10 en dos pruebas o más presentaban deterioro cognoscitivo.

Actividades básicas de la vida diaria (ABVD). Se utilizaron las actividades consideradas en el índice de Katz (18) para evaluar la capacidad de realizar sin ayuda seis funciones básicas: bañarse, vestirse, usar el sanitario, trasladarse dentro del hogar, controlar los esfínteres y alimentarse. Las ABVD se analizaron de manera individual y en conjunto. Se consideró que la pérdida de una función reflejaba cierto grado de dependencia, equivalente a la resta de un punto de una puntuación máxima de seis.

Actividades instrumentales de la vida diaria (AIVD). Se utilizaron las actividades consideradas en el índice de Lawton y Brody (19) para evaluar la capacidad de realizar sin ayuda ocho funciones: preparar los alimentos, hacer los quehaceres o las reparaciones en casa, lavar la ropa, tomar los medicamentos, desplazarse con el uso de medios de transporte, ir de compras, administrar el dinero y utilizar el teléfono. Las AIVD se analizaron de manera individual y en conjunto. Se consideró que la pérdida de una función reflejaba cierto grado de dependencia, equivalente a la resta de un punto de una puntuación máxima de 8 en las mujeres y de 5 en los hombres (no se tomaron en cuenta tres actividades por considerarlas más propias de las mujeres en la cultura y el contexto en que se desarrolló la investigación).

Otras actividades. Como variables dicotómicas se exploró la dificultad para realizar otras actividades: caminar una o varias calles, subir uno o varios pisos por las escaleras, levantarse de una silla, levantar los brazos y las piernas y levantar una moneda de una mesa.

Índice de masa corporal y hábitos de vida. Se calculó el índice de masa corporal (peso en kilogramos entre la talla en metros cuadrados). También se exploró, como variables dicotómicas, si había sido fumador (haber fumado más de 100 cigarrillos en su vida) y si consumía bebidas alcohólicas.

\section{Análisis estadístico}

Las características de los participantes se presentan como la media y su desviación estándar en las variables continuas y como frecuencia y porcentaje en las variables categóricas. Inicialmente se identificaron las variables asociadas significativamente con el autoinforme de HTNT (variable dependiente). Para tal propósito, se utilizaron las pruebas de la $t$ de Student y de la ji al cuadrado, según el tipo de variable. Posteriormente, las variables que resultaron estadísticamente significativas se probaron por separado en modelos de regresión logística simple para demostrar su asociación independiente con la HTNT. Las variables que mantuvieron su significación se em- plearon para construir otros modelos de regresión logística con múltiples variables agrupadas según su categoría (sociodemográficas, de hábitos de vida, de actividades de la vida diaria, etc.). Finalmente, se realizó un análisis de regresión logística multifactorial con las variables que resultaron significativas dentro de cada grupo para comprobar su asociación independiente con el autoinforme de HTNT. Este último modelo se ajustó por otras variables confusoras.

Mediante el análisis de HosmerLemeshow y de los valores residuales se comprobó el buen ajuste del modelo y el cumplimiento de los postulados de base necesarios para la validez de la regresión logística (20). Se calcularon la razón de posibilidades (odds ratio, OR) y los intervalos de confianza de 95\% (IC95\%). Se utilizó un nivel de significación estadística $P<0,05$, excepto en la construcción del modelo final, donde se empleó un nivel más riguroso $(P<0,01)$. Para todos los análisis se utilizó el paquete estadístico SPSS para Windows ${ }^{\circledR}$ versión 13.0 (SPSS Inc., Chicago, IL).

El ENASEM recibió la aprobación del Comité de Ética de la Universidad de Pensilvania, Estados Unidos.

\section{RESULTADOS}

De 15402 entrevistas realizadas en el ENASEM, 5551 (36,0\%) participantes declararon haber tenido al menos un diagnóstico médico de HTA, de ellos 2029 tenían más de 65 años y conformaron la muestra de estudio. De esta muestra, 1264 (62,3\%) eran mujeres y 765 (37,7\%) eran hombres. La edad promedio fue de 72,9 $\pm 6,6$ años (mínima: 65; máxima: 105). En el cuadro 1 se presentan las características generales de la muestra estudiada.

De los encuestados de la muestra, 437 (21,5\%) declararon no seguir tratamiento alguno para controlar la HTA a pesar de haber recibido el diagnóstico médico correspondiente, 1584 (78,1\%) afirmaron seguir un tratamiento y 8 $(0,4 \%)$ no respondieron a esa pregunta. No hubo diferencias significativas entre los participantes que declararon 
tomar algún medicamento antihipertensivo y los que no los declararon en cuanto a la presencia de deterioro cognoscitivo $(P=0,73)$ y otras enfermedades crónicas, como la enfermedad pulmonar obstructiva crónica $(P=0,76)$, la enfermedad vascular cerebral $(P=0,29)$ y la artritis $(P=0,15)$.

Los participantes que informaron seguir algún tratamiento antihipertensivo presentaron con menor frecuencia debilidad visual $(47,7 \%$ frente a $53,1 \%$; $P=0,04)$ y síntomas depresivos $(48,1 \%$ frente a $53,5 \% ; P=0,05)$, así como un mayor número de enfermedades crónicas $(1,9$ frente a 1,$7 ; P<0,01)$ que los que no seguían ningún tratamiento para controlar la HTA (cuadro 1). Las personas que informaron no tomar medicamentos antihipertensivos manifestaron con mayor frecuencia no tener dinero para adquirir los medicamentos que las que los tomaban $(21,3 \%$ frente a $15,7 \% ; P<0,01)$. No se encontraron diferencias significativas entre ambos grupos en cuanto a presentar dificultades para salir de compras $(P=0,06)$, administrar el dinero $(P=0,84)$ o tomar los medicamentos $(P=0,84)$ (cuadro 2$)$. Tampoco hubo diferencias con respecto a la dificultad para realizar al menos dos $\operatorname{ABVD}(P=0,42)$, caminar una o varias calles $(P=0,69)$, levantarse de una silla $(P=0,26)$, levantar los brazos $(P=0,86)$ y levantar una moneda de una mesa $(P=0,31)$.

Según el análisis logístico simple, no se encontró asociación entre la edad (OR = 1,00; IC95\%: 0,99 a 1,02; $P=0,65$ ) y el sexo (OR = 1,12; IC95\%: 0,90 a 1,39; $P=0,31)$ con la HTNT. Trabajar sin recibir una paga $(\mathrm{OR}=1,37$; IC95\%: $1,09$ a 1,$72 ; P<0,01)$, presentar debilidad visual (OR = 1,24; IC95\%: 1,00 a $1,54 ; P=0,04)$ y no comprar los medicamentos por falta de dinero $(\mathrm{OR}=1,45 ; \mathrm{IC} 95 \%$ : 1,10 a 1,92; $P<0,01)$, entre otras variables, se asociaron individualmente con el autoinforme de HTNT (cuadro 3).

El análisis multifactorial ajustado por posibles variables confusoras (edad, sexo, síntomas depresivos y deterioro cognoscitivo) mostró que solo la baja escolaridad (OR ajustada = 1,70; IC95\%: 1,10 a 2,64; $P=0,02$ para la escolaridad de 1 a 6 años y OR ajustada $=3,32$;

CUADRO 1. Características sociodemográficas y de salud de la muestra estudiada. ${ }^{\text {a }}$ Estudio Nacional sobre Salud y Envejecimiento en México, 2001

\begin{tabular}{lccr}
\hline \multicolumn{1}{c}{ Variable } & $\begin{array}{c}\text { Participantes con } \\
\text { tratamiento }(\%)^{\mathrm{b}}\end{array}$ & $\begin{array}{c}\text { Participantes sin } \\
\text { tratamiento }(\%)\end{array}$ & $P^{c}$ \\
\hline Edad & $72,8 \pm 6,6$ & $73,0 \pm 6,6$ & 0,65 \\
Hombres & $589(37,2)$ & $174(39,8)$ & 0,31 \\
Mujeres & $995(62,8)$ & $263(60,2)$ & \\
Escolaridad $\geq 7$ años & $235(14,9)$ & $35(8,0)$ & $<0,01$ \\
Tiene pareja & $797(51,9)$ & $217(50,6)$ & 0,64 \\
Trabaja sin recibir una paga & $415(26,5)$ & $144(33,1)$ & $<0,01$ \\
Tiene un seguro de vida & $123(10,4)$ & $25(7,1)$ & 0,07 \\
Mala autopercepción de su salud & $447(31,3)$ & $101(25,5)$ & 0,16 \\
Tiene diabetes mellitus & $423(26,7)$ & $68(15,6)$ & $<0,01$ \\
Tiene cardiopatía isquémica & $155(9,8)$ & $24(5,5)$ & $<0,01$ \\
Tiene enfermedad pulmonar obstructiva crónica & $160(10,1)$ & $42(9,6)$ & 0,76 \\
Tuvo enfermedad vascular cerebral & $109(6,9)$ & $24(5,5)$ & 0,29 \\
Tiene artritis & $526(33,2)$ & $129(29,7)$ & 0,15 \\
Número de enfermedades crónicas que padece & $1,9 \pm 0,9$ & $1,7 \pm 0,8$ & $<0,01$ \\
Tiene síntomas depresivos & $687(48,1)$ & $212(53,5)$ & 0,05 \\
Presenta debilidad visual & $755(47,7)$ & $232(53,1)$ & 0,04 \\
Presenta deterioro cognoscitivo & $446(32,5)$ & $127(33,4)$ & 0,73 \\
Índice de masa corporal (kg/m $\left.{ }^{2}\right)$ & $27,1 \pm 5,1$ & $26,5 \pm 5,0$ & 0,08 \\
Ha fumado más de 100 cigarrillos en su vida & $129(19,2)$ & $50(25,9)$ & 0,04 \\
Consume bebidas alcohólicas & $297(21,4)$ & $112(28,4)$ & $<0,01$ \\
\hline
\end{tabular}

a En total 1584 participantes informaron que seguían algún tratamiento, 437 dijeron que no lo seguían y 8 no respondieron esa pregunta ( 6 mujeres y 2 hombres). La cuantificación de cada variable se realizó a partir del número de respuestas recibidas.

${ }^{b}$ Excepto en la edad, el número de enfermedades crónicas y el índice de masa corporal, en que se empleó la media \pm desviación estándar.

c Según las pruebas de la $t$ de Student o de la ji al cuadrado, según el tipo de variable. Nivel de significación $P<0,05$.

CUADRO 2. Características funcionales de los participantes. ${ }^{a}$ Estudio Nacional sobre Salud y Envejecimiento en México, 2001

\begin{tabular}{|c|c|c|c|}
\hline Variable & $\begin{array}{l}\text { Participantes con } \\
\text { tratamiento }(\%)\end{array}$ & $\begin{array}{l}\text { Participantes sin } \\
\text { tratamiento (\%) }\end{array}$ & $P^{\mathrm{b}}$ \\
\hline No compra medicamentos por falta de dinero & $222(15,7)$ & $84(21,3)$ & $<0,01$ \\
\hline Tiene problemas físicos para tomar & $87(6.2)$ & $23(5.9)$ & 0.84 \\
\hline Tiene dificultades para salir de compras & $200(15,3)$ & $42(11,4)$ & 0,06 \\
\hline Tiene dificultades para administrar el dinero & $76(5,4)$ & $22(5,7)$ & 0,84 \\
\hline Piensa que el ejercicio y la dieta son buenos & $1229(87,9)$ & $345(89,4)$ & 0,43 \\
\hline Tiene incontinencia urinaria & $176(12,4)$ & $70(17,7)$ & $<0,01$ \\
\hline Tiene dificultad para caminar $\geq 1$ calle & $633(45,3)$ & $172(44,3)$ & 0,69 \\
\hline Tiene dificultad para levantarse de una silla & $627(43,9)$ & $161(40,8)$ & 0,26 \\
\hline Tiene dificultad para levantar los brazos & $305(21,4)$ & $83(21,0)$ & 0,86 \\
\hline $\begin{array}{l}\text { Tiene dificultad para levantar una moneda } \\
\text { de la mesa }\end{array}$ & $175(12,3)$ & $41(10,4)$ & 0,31 \\
\hline Tiene dependencia para realizar $\geq 2 \mathrm{ABVD}^{\mathrm{C}}$ & $111(7,6)$ & $23(5,8)$ & 0,42 \\
\hline Tiene dependencia para realizar $\geq 2$ AIVD $^{d}$ & $191(13,4)$ & $33(8,4)$ & $<0,01$ \\
\hline
\end{tabular}

${ }^{a}$ En total 1584 participantes informaron que seguían algún tratamiento, 437 dijeron que no lo seguían y 8 no respondieron esa pregunta (6 mujeres y 2 hombres). La cuantificación de cada variable se realizó a partir del número de respuestas recibidas. b Según las pruebas de la $t$ de Student o de la ji al cuadrado, según el tipo de variable. Nivel de significación $P<0,05$. ${ }^{c}$ ABVD: actividades básicas de la vida diaria.

${ }^{d}$ AIVD: actividades instrumentales de la vida diaria.

IC95\%: 2,10 a 5,24; $P<0,01$, para los no escolarizados), consumir bebidas alcohólicas (OR ajustada = 1,52; IC95\%: 1,14 a 2,$03 ; P=0,01)$ y padecer de inconti- nencia urinaria $(\mathrm{OR}$ ajustada $=1,61$; IC95\%: 1,15 a 2,26 ; $P<0,01$ ) resultaron asociadas independientemente con la HTNT (cuadro 4). Los resultados de ese 
CUADRO 3. Análisis de regresión logística simple de la hipertensión no tratada en los adultos mayores. Estudio Nacional sobre Salud y Envejecimiento en México, 2001

\begin{tabular}{|c|c|c|c|c|c|}
\hline Variable & Coeficiente & Error & $\mathrm{OR}^{\mathrm{a}}$ & IC95\% ${ }^{b}$ & $P^{c}$ \\
\hline Edad (años) & 0,004 & 0,008 & 1,00 & $0,99-1,02$ & 0,65 \\
\hline Sexo (hombres vs. mujeres) & 0,111 & 0,111 & 1,12 & $0,90-1,39$ & 0,31 \\
\hline \multicolumn{6}{|l|}{ Escolaridad } \\
\hline$\geq 7$ años & - & - & 1,00 & - & \\
\hline De 1 a 6 años & 0,454 & 0,197 & 1,57 & $1,07-2,32$ & 0,02 \\
\hline$<1$ año & 1,010 & 0,200 & 2,75 & $1,86-4,06$ & $<0,01$ \\
\hline Trabajar sin recibir una paga & 0,315 & 0,117 & 1,37 & $1,09-1,72$ & $<0,01$ \\
\hline Tener diabetes mellitus & $-0,683$ & 0,144 & 0,50 & $0,38-0,67$ & $<0,01$ \\
\hline Tener cardiopatía isquémica & $-0,621$ & 0,226 & 0,54 & $0,34-0,83$ & $<0,01$ \\
\hline Número de enfermedades crónicas & $-0,309$ & 0,068 & 0,73 & $0,64-0,84$ & $<0,01$ \\
\hline Presentar síntomas depresivos & 0,219 & 0,114 & 1,24 & $0,99-1,55$ & 0,05 \\
\hline Presentar debilidad visual & 0,217 & 0,108 & 1,24 & $1,00-1,54$ & 0,04 \\
\hline Usar lentes & $-0,596$ & 0,112 & 0,55 & $0,44-0,69$ & $<0,01$ \\
\hline Haber fumado más de 100 cigarrillos en su vida & 0,385 & 0,191 & 1,47 & $1,01-2,14$ & 0,04 \\
\hline Consumir bebidas alcohólicas & 0,375 & 0,129 & 1,45 & $1,13-1,88$ & $<0,01$ \\
\hline No comprar medicamentos por falta de dinero & 0,372 & 0,143 & 1,45 & $1,10-1,92$ & $<0,01$ \\
\hline Tener incontinencia urinaria & 0,420 & 0,154 & 1,52 & $1,13-2,06$ & $<0,01$ \\
\hline \multicolumn{6}{|l|}{ Tener dependencia para realizar AIVD ${ }^{d}$} \\
\hline Sin dependencia & - & - & 1 & - & - \\
\hline En una actividad & $-0,328$ & 0,218 & 0,72 & $0,47-1,10$ & 0,13 \\
\hline En $\geq 2$ actividades & $-0,556$ & 0,199 & 0,57 & $0,39-0,85$ & $<0,01$ \\
\hline
\end{tabular}

a OR: razón de posibilidades (odds ratios).

b IC95\%: intervalo de confianza de $95 \%$.

c Según la prueba de Wald. Nivel de significación $P<0,05$.

${ }^{d}$ AIVD: Actividades instrumentales de la vida diaria.

CUADRO 4. Análisis multifactorial de regresión logística de la hipertensión no tratada. ${ }^{a}$ Estudio Nacional sobre Salud y Envejecimiento en México, 2001

\begin{tabular}{|c|c|c|c|c|c|}
\hline Variable & Coeficiente & Error & $\mathrm{OR}^{\mathrm{b}}$ & $\operatorname{IC95\% } \%^{c}$ & $P^{d}$ \\
\hline \multicolumn{6}{|l|}{ Escolaridad } \\
\hline$\geq 7$ años & - & - & 1 & - & - \\
\hline De 1 a 6 años & 0,531 & 0,225 & 1,70 & $1,10-2,64$ & 0,02 \\
\hline$<1$ año & 1,199 & 0,233 & 3,32 & $2,10-5,24$ & $<0,01$ \\
\hline Consumir bebidas alcohólicas & 0,417 & 0,148 & 1,52 & $1,14-2,03$ & 0,01 \\
\hline Tener incontinencia urinaria & 0,477 & 0,173 & 1,61 & $1,15-2,26$ & $<0,01$ \\
\hline Tener diabetes mellitus & $-0,713$ & 0,168 & 0,50 & $0,35-0,68$ & $<0,01$ \\
\hline Tener cardiopatía isquémica & $-0,648$ & 0,283 & 0,53 & $0,30-0,91$ & 0,02 \\
\hline \multicolumn{6}{|l|}{ Tener dependencia para realizar AIVD } \\
\hline Sin dependencia para realizar AIVD & - & - & 1 & - & - \\
\hline En una actividad & $-0,651$ & 0,240 & 0,83 & $0,52-1,34$ & 0,44 \\
\hline En $\geq 2$ actividades & $-0,554$ & 0,216 & 0,57 & $0,38-0,89$ & 0,01 \\
\hline
\end{tabular}

a Los resultados están ajustados por la edad, el sexo y la presencia de síntomas depresivos y de deterioro cognoscitivo.

b OR: razón de posibilidades (odds ratios).

c IC95\%: intervalo de confianza de $95 \%$.

d Según la prueba de Wald. Nivel de significación $P<0,05$.

e AIVD: actividades instrumentales de la vida diaria.

modelo indican que tener diabetes mellitus (OR ajustada =0,50; IC95\%: 0,35 a 0,$68 ; P<0,01)$ y cardiopatía isquémica (OR ajustada =0,53; IC95\%: 0,30 a 0,$91 ; P=0,02)$ y necesitar ayuda en al menos dos AIVD (OR ajustada = 0,57; IC95\%: 0,38 a 0,$89 ; P=0,01)$ también se asociaron inversamente con la declaración de no tomar medicamento alguno para controlar la HTA.

Los resultados del análisis de residuos y de la prueba de HosmerLemeshow demostraron el buen ajuste del modelo $(P>0,05)$.

\section{DISCUSIÓN}

La falta de un control adecuado en la tensión arterial de los pacientes hipertensos, que requiere por lo general de medicamentos, contribuye al avance de la enfermedad y a la apari- 
ción de sus complicaciones. Este estudio demuestra que factores muy diversos están asociados con la conducta de los adultos mayores de no cumplir con el tratamiento prescrito para la HTA. La identificación de esos factores constituye un recurso útil para el médico y le permitirá prever qué pacientes necesitarán alguna intervención especial para mejorar o asegurar su adecuada adhesión al tratamiento.

Un mensaje clave del séptimo informe del Comité Nacional Conjunto para la Prevención, Detección, Evaluación y Tratamiento de la Hipertensión Arterial, de los Estados Unidos, es que la HTA, en particular la sistólica, es un importante factor de riesgo de la enfermedad cardiovascular, especialmente en los adultos mayores (21). Esto obliga a llevar un control más activo de la tensión arterial. Sin embargo, a pesar de una adecuada prescripción, los médicos no cuentan con los elementos necesarios para reconocer la falta de adhesión al tratamiento, por lo que las intervenciones para mejorarla han tenido resultados muy variados y pueden resultar complejas y costosas (11, 22, 23). Está claro que el pleno beneficio del tratamiento antihipertensivo solamente se consigue si los pacientes lo siguen de forma adecuada (11), por consiguiente, las características del enfermo deben tomarse en cuenta al decidir el tipo de régimen antihipertensivo que se prescribe.

$\mathrm{Si}$ bien el sexo masculino y el nivel socioeconómico bajo son factores de predicción ya conocidos de la mala adhesión al tratamiento farmacológico, estos resultados no han sido consistentes (24). La Asociación Estadounidense del Corazón informó de distintos factores asociados con la falta de adhesión del paciente al tratamiento, como la edad, el estado marital y la personalidad del enfermo (25). En la muestra estudiada no hubo diferencias en cuanto a la edad y el sexo en este sentido, sin embargo, el autoinforme de "no comprar los medicamentos por falta de dinero" -que puede considerarse un reflejo de la insuficiencia económicafue más frecuente en los participantes que declararon no seguir ningún tratamiento antihipertensivo. A pesar de que las enfermedades psiquiátricas - principalmente la depresión- y el deterioro cognoscitivo se han asociado a la falta de apego a los tratamientos farmacológicos (26), estas variables no mostraron una asociación significativa independiente con el autoinforme de HTNT en la población estudiada. Por otro lado, y a diferencia de los resultados de las fases I y II de la Tercera Encuesta Nacional de Salud y Nutrición (NAHNES III) en los Estados Unidos (8), la baja escolaridad en la población estudiada sí estuvo asociada con el autoinforme de HTNT, lo que confirma investigaciones anteriores (27).

Los adultos mayores deben recibir un trato diferente al de la población general para el control de su HTA, ya que al prescribir el tratamiento antihipertensivo se deben tomar en cuenta las enfermedades crónicas concomitantes, frecuentes en las personas de este grupo de edad. No obstante, el estado funcional casi siempre es pasado por alto en el momento de elegir el tratamiento y no se tienen en cuenta la debilidad visual, la depresión y los problemas de la memoria, que tienen una clara influencia en el cumplimiento estricto del tratamiento (28). Se debe prestar especial atención a la incontinencia urinaria, pues el tratamiento de elección para controlar la HTA no complicada se basa en diuréticos, solos o combinados (21). Si bien los diuréticos presentan ventajas para el control de la HTA del adulto mayor (por su bajo costo y fácil administración), estos fármacos pueden producir o descompensar la incontinencia urinaria, lo cual puede provocar la falta de adhesión del paciente al tratamiento prescrito (29). Con frecuencia, los médicos pasan por alto este y otros inconvenientes del tratamiento antihipertensivo, lo que da como resultado un insuficiente control de la tensión arterial.

El consumo de bebidas alcohólicas se ha asociado con la dificultad para controlar la tensión arterial (3). Sin embargo, la asociación encontrada en este estudio requiere un análisis cuidadoso ya que se consideró el consumo de bebidas alcohólicas en un sentido muy amplio (abarcaba desde los que tomaban bebidas alcohólicas esporádica- mente hasta los posibles alcohólicos) y no obstante resultó asociado clara e independientemente con la HTNT.

Es necesario considerar también que, independientemente de la forma en que el médico explique los beneficios de seguir adecuadamente el tratamiento antihipertensivo, será el paciente el responsable final de tomar o no sus medicamentos. En este sentido, no es sorprendente que los pacientes con dependencia para realizar más de dos AIVD informen una mejor adhesión al tratamiento para controlar la HTA, ya que de alguna manera la supervisión que tienen de una segunda persona para administrar el dinero, cocinar y otras actividades cotidianas, se refleja en una mejor adhesión al tratamiento. Sin embargo, en esta muestra no se encontraron diferencias significativas en cuanto a la capacidad física para tomar sus propios fármacos entre los participantes que informaron tomar algún antihipertensivo y los que informaron que no lo tomaban.

El tiempo de evolución de la enfermedad y su naturaleza son determinantes para la adhesión al tratamiento farmacológico. Los pacientes con enfermedades crónicas consideradas graves, como la cardiopatía isquémica o la diabetes mellitus, parecen estar más dispuestos a controlarlas. En los pacientes con múltiples enfermedades crónicas, la adhesión al tratamiento para alguna de sus enfermedades más graves puede verse reflejado en un adecuado apego al tratamiento de las otras $(30,31)$. Involucrar al paciente en el control de su enfermedad, con actividades como la autovigilancia de la tensión arterial, pudiera servir para promover su adecuada adhesión al tratamiento (32).

Este estudio tiene varias limitaciones. Primero, el diseño transversal empleado no permite establecer la dirección causal de las asociaciones encontradas, por lo que para ello habrá que diseñar estudios longitudinales. Segundo, la información se obtuvo mediante autoinformes, sin la verificación clínica del diagnóstico de HTA y como el seguimiento de un tratamiento hipertensivo era la variable dependiente, no se pudo incorporar como pregunta 
directa en el autoinforme. En los grandes estudios epidemiológicos muchas veces no es viable (por problemas logísticos o económicos) medir algunas variables - como el estado funcionalo que un profesional evalúe individualmente a los participantes para corroborar la presencia o gravedad de las enfermedades y los síntomas informados. Sin embargo, los autoinformes pueden servir para evaluar de forma simple y efectiva el apego a un tratamiento (33), aun cuando puedan estar distorsionados por el paciente. No obstante, las variables analizadas en este estudio mediante el modelo multifactorial resultaron significativas, independientemente del efecto de los síntomas depresivos y del deterioro cognoscitivo de los participantes. Tercero, en esta muestra de adultos mayores, casi $72 \%$ dijeron seguir algún tratamiento antihipertensivo, lo que puede tratarse de una sobrestimación. Según la ENSA 2000, menos de la mitad de las personas con HTA diagnosticada toma algún medicamento y de estos, solo alrededor de $20 \%$ mantiene la HTA controlada (5). En el presente estudio no se comprobó si los adultos mayores que declararon tomar algún tratamiento mantenían un ade- cuado control de la tensión arterial o si los medicamentos empleados estaban prescritos por un médico. La tendencia a dar una respuesta "socialmente correcta", como es responder afirmativamente a la pregunta sobre si sigue el tratamiento para una enfermedad diagnosticada por un médico, pudo originar un sesgo en la respuesta de los participantes.

En este estudio no se analizaron algunas variables que pudieran estar asociadas con la HTNT, como el tipo de relación existente entre el médico y el paciente y la complejidad del tratamiento prescrito. Al parecer, una medicación simple (monodosis) y adecuada (sin efectos adversos importantes) puede contribuir a mejorar la adhesión al tratamiento, incluso en los pacientes que tienen problemas de memoria o son analfabetos (34-36).

Como conclusión, estos resultados permiten afirmar que la HTNT es un problema frecuente e importante en los adultos mayores en México. La baja escolaridad, el consumo de bebidas alcohólicas y la incontinencia urinaria se asociaron significativamente con la declaración de HTNT. Los médicos deben tomar en cuenta estos trastornos al prescribir o modificar el trata- miento antihipertensivo, ya que el uso de medicamentos para controlar la HTA puede ser más eficaz cuando el paciente está motivado y participa en la decisión médica.

Para aumentar la motivación y participación de los adultos mayores hipertensos en las decisiones clínicas se debe mejorar la relación médicopaciente y elevar su confianza en el tratamiento, por lo que el modelo de atención autoritario debe sustituirse por uno más dinámico. Esto es particularmente importante en el tratamiento de los adultos mayores que se muestran renuentes a cambiar su estilo de vida y a aceptar esta nueva etapa. Se deben emprender estudios longitudinales para establecer la dirección causal de las asociaciones establecidas en este estudio.

Agradecimientos. El ENASEM recibió apoyo del Instituto Nacional de Envejecimiento de los Institutos Nacionales de Salud de los Estados Unidos mediante una beca de investigación. Además, recibió el apoyo de investigadores de las Universidades de Pennsylvania, Maryland y Wisconsin, y del Instituto Nacional de Estadística, Geografía e Informática de México.

\section{REFERENCIAS}

1. Estados Unidos Mexicanos, Secretaría de Salud. Estadísticas de egresos hospitalarios del sector público del Sistema Nacional de Salud, 2001. Salud Publica Mex. 2003;45: 310-27.

2. Instituto Nacional de Estadística, Geografía e Informática. Los adultos mayores en México. Perfil sociodemográfico al inicio del siglo XXI [sitio en Internet]. México: INEGI; 2005. Hallado en: http:/ /www.inegi.gob.mx/ prod_serv/contenidos/espanol/bvinegi/ productos/censos/poblacion/adultos mayores/Adultos_mayores_web2.pdf. Acceso el 29 de enero de 2008

3. Joint National Committee on Prevention, Detection, Evaluation, and Treatment of High Blood Pressure. The sixth report of the Joint National Committee on Prevention, Detection, Evaluation, and Treatment of High Blood Pressure. Arch Intern Med. 1997;157: 2413-46.

4. Vasan RS, Beiser A, Seshadri S, Larson MG, Kannel WB, D'Agostino RB, et al. Residual lifetime risk for developing hypertension in middle-aged women and men: The Framing- ham Heart Study. J Am Med Assoc. 2002;287: 1003-10.

5. Velázquez MO, Rosas PM, Lara EA, Pastelín HG. Hipertensión arterial en México: resultados de la Encuesta Nacional de Salud (ENSA) 2000. Arch Cardiol Mex. 2002;72:71-84.

6. Fields LE, Burt VL, Cutler JA, Hugher J, Roccella EJ, Sorlie P. The burden of adult hypertension in the United States 1999 to 2000: a rising tide. Hypertension. 2004;44;398-404.

7. Goldman L, Cook EF. The decline in ischemic heart disease mortality rates. An analysis of the comparative effects of medical interventions and changes in lifestyle. Ann Intern Med. 1984;101:825-36.

8. Hyman DJ, Pavlik VN. Characteristics of patients with uncontrolled hypertension in the United States. N Engl J Med. 2001;345:479-86.

9. Senst BL, Achusim LE, Genest RP, Cosentino LA, Ford CC, Little JA, et al. Practical approach to determining costs and frequency of adverse drug events in a health care network. Am J Health Syst Pharm. 2001;58:1126-32.

10. Lewington S, Clarke R, Qizilbash N, Peto R, Collins R. Age-specific relevance of usual blood pressure to vascular mortality: a metaanalysis of individual data for one million adults in 61 prospective studies. Lancet. 2002; 360:1903-13.

11. Osterberg L, Blaschke T. Adherence to medication. N Engl J Med. 353:487-97.

12. Berlowitz DR, Ash AS, Hickey EC, Friedman RH, Glickman M, Kader B, et al. Inadequate management of blood pressure in a hypertensive population. N Engl J Med. 1998;339: 1957-63.

13. Oliveria SA, Lapuerta P, McCarthy BD, L'Italien GJ, Berlowitz DR, Asch SM. Physician-related barriers to the effective management of uncontrolled hypertension. Arch Intern Med. 2002;162:413-20.

14. Estudio Nacional sobre Salud y Envejecimiento en México (ENASEM) [sitio en Internet]. México, D.F.: Universidad de Pennsylvania. Hallado en: http://www.ssc.upenn.edu/ mhas/. Acceso el 29 de enero de 2008.

15. Instituto Nacional de Estadística, Geografía e Informática. II Conteo de Población y Vivienda [sitio en Internet]. México, D.F.: INEGI; 2005. Hallado en: http://www.inegi. 
gob.mx/est/contenidos/espanol/sistemas/ conteo2005/datos/00/excel/cpv00_viv_11. xls. Acceso el 28 de febrero de 2008.

16. Aguilar-Navarro SG, Fuentes-Cantú A, ÁvilaFunes JA, García-Mayo EJ. Validity and reliability of the screening questionnaire for geriatric depression used in the Mexican Health and Age Study. Salud Publica Mex. 2007;49: 256-62.

17. Glosser G, Wolfe N, Albert ML, Lavine L, Steele JC, Calne DB, et al. Cross-cultural cognitive examination: validation of a dementia screening instrument for neuroepidemiological research. J Am Geriatr Soc. 1993;41:931-9.

18. Katz S, Akpom CA. 12 Index of ADL. Med Care. 1976;14:116-8.

19. Lawton MP, Brody EM. Assessment of older people: self-maintaining and instrumental activities of daily living. Gerontologist. 1969;9: 179-86.

20. Lemeshow S, Hosmer DW Jr. A review of goodness of fit statistics for use in the development of logistic regression models. Am J Epidemiol. 1982;115:92-106.

21. Chobanian AV, Bakris GL, Black HR, Cushman WC, Green LA, Izzo JL Jr, et al. The seventh report of the Joint National Committee on Prevention, Detection, Evaluation, and Treatment of High Blood Pressure. J Am Med Assoc. 2003;289:2560-72.

22. Burnier M. Long-term compliance with antihypertensive therapy: another facet of chronotherapeutics in hypertension. Blood Press Monit. 2000;5:S31-4.
23. Haynes RB, McDonald H, Garg AX, Montague $\mathrm{P}$. Interventions for helping patients to follow prescriptions for medications. Cochrane Database Syst Rev. 2002;2:CD000011.

24. Balkrishnan R. Predictors of medication adherence in the elderly. Clin Ther. 1998;20: 764-71.

25. Miller NH, Hill M, Kottke T, Ockene IS. The multilevel compliance challenge: recommendations for a call to action. A statement for healthcare professionals. Circulation. 1997;95: 1085-90.

26. Stilley CS, Sereika S, Muldoon MF, Ryan CM, Dunbar-Jacob J. Psychological and cognitive function: predictors of adherence with cholesterol lowering treatment. Ann Behav Med. 2004;27:117-24.

27. Lerman I. Adherence to treatment: the key for avoiding long-term complications of diabetes. Arch Med Res. 2005;36:300-6.

28. Rodríguez-Mañas L, Zajarías-Kursckansky A. Hipertensión arterial. En: Gutiérrez-Robledo LM, d'Hyver C, ed. Geriatría. 1. ${ }^{a}$ ed. México, D.F.: Manual Moderno; 2006. Pp. 273-9.

29. Fincke BG, Miller DR, Spiro A 3rd. The interaction of patient perception of overmedication with drug compliance and side effects. J Gen Intern Med. 1998;13:182-5.

30. Piette JD, Richardson C, Valenstein M. Addressing the needs of patients with multiple chronic illnesses: the case of diabetes and depression. Am J Manag Care. 2004;10:152-62.

31. Blackburn DF, Dobson RT, Blackburn JL, Wilson TW, Stang MR, Semchuk WM. Adherence to statins, beta-blockers and angiotensinconverting enzyme inhibitors following a first cardiovascular event: a retrospective cohort study. Can J Cardiol. 2005;21:485-8.

32. Feldman R, Bacher M, Campbell N, Drover A, Chockalingam A. Adherence to pharmacologic management of hypertension. Can J Public Health. 1998;89:I16-8.

33. Haynes RB, Taylor DW, Sackett DL, Gibson ES, Bernholz CD, Mukherjee J. Can simple clinical measurements detect patient noncompliance? Hypertension. 1980;2:757-64.

34. Eisen SA, Miller DK, Woodward RS, Spitznagel E, Przybeck TR. The effect of prescribed daily dose frequency on patient medication compliance. Arch Intern Med. 1990;150: $1881-4$.

35. Leenen FH, Wilson TW, Bolli P, Larochelle $\mathrm{P}$, Myers M, Handa SP, et al. Patterns of compliance with once versus twice daily antihypertensive drug therapy in primary care: a randomized clinical trial using electronic monitoring. Can J Cardiol. 1997;13:914-20.

36. Borzecki AM, Oliveria SA, Berlowitz DR. Barriers to hypertension control. Am Heart J. 2005;149:785-94.

Manuscrito recibido el 9 de mayo de 2007. Aceptado para publicación, tras revisión, el 19 de noviembre de 2008.
ABSTRACT

Factors associated with untreated hypertension among older adults: results of the Mexican Health and Aging Study, 2001
Objective. To determine factors associated with failure to adhere to treatment for diagnosed hypertension among a representative sample of older Mexican adults living in the community.

Methods. A cross-sectional study of 2029 individuals 65 years of age or older with diagnosed hypertension who participated in the Mexican Health and Aging Study, carried out during the summer of 2001. The survey collected information on several demographics (age, sex, schooling, whether living alone, and employment status, among others), any chronic illnesses, symptoms of depression, cognitive deterioration, body mass index, smoking, alcohol consumption, and difficulty performing basic and instrumental activities of daily living. Simple and multifactorial logistical regression analyses were used to evaluate the association among the study variables and self-reported untreated high blood pressure.

Results. Of the 2029 participants, $437(21.5 \%)$ reported not following any treatment whatsoever for controlling their hypertension; 1584 (78.1\%) affirmed they were complying with treatment; and $8(0.4 \%)$ did not respond to this question. The multifactorial analysis adjusted for confounding variables (age, sex, symptoms of depression, and cognitive deterioration) showed that only a low number of years of schooling (adjusted odds ratio $[\mathrm{OR}]=1.70 ; 95 \%$ confidence interval $[95 \% \mathrm{CI}]: 1.10-2.64 ; P=0.02$ for 1-6 years of schooling and adjusted OR $=3.32 ; 95 \% \mathrm{CI}: 2.10-5.24 ; P<0.01$ for no schooling), alcohol consumption (adjusted OR $=1.52 ; 95 \% C I$ : $1.14-2.03 ; P=0.01$ ), and urinary incontinence (adjusted $\mathrm{OR}=1.61 ; 95 \% \mathrm{CI}$ : 1.15-2.26; $P<0.01$ ) were independently associated with hypertension.

Conclusions. Hypertension is a common and important issue among older adults in Mexico. To obtain better medication compliance, doctors prescribing or modifying hypertension treatment should taken into account whether or not the patient suffers from urinary incontinence, consumes alcohol, and/or has a low level of schooling.

Key words Aged, hypertension control, patient compliance, Mexico. 